destruction, proinflammatory cytokine release. TWEAK and Fn14 receptor interaction has an important role in pathogenesis of atherosclerosis. Our aim was to evaluate subclinical atherosclerosis and its association with serum STWEAK, Fn14 and CD163 levels in RA patients.

Methods: One hundred Rheumatoid Arthritis (RA), 50 Spondyloarthritis (SpA) patients and 50 healthy controls $(\mathrm{HC})$ were included in the study. Serum soluble TWEAK, CD163 and Fn14 levels were measured by ELISA tecnique. Subclinical atherosclerosis was evaluated by echocardiography, which includes carotis intima media thickness (cIMT), aortic strain, stiffness and elasticity.

Results: In RA group 73 (73\%) patients were using DMARD's and 27 (27\%) were biological drugs, the mean DAS28 score was $3.49 \pm 1.31$. At the end of the study, TWEAK levels were found significantly lower (RA; $942.4 \pm 305.9$, AS; $1087.2 \pm 311.0$ and $\mathrm{HC} ; 1061.3 \pm 402.8 \mathrm{pg} / \mathrm{ml}, \mathrm{p}=0.05)$, and $\mathrm{Fn} 14$ levels were significantly higher (RA; $509.4 \pm 861.4$, AS; $163.5 \pm 249.0$ and $\mathrm{HC} ; 91.5 \pm 67.9 \mathrm{pg} / \mathrm{ml}, \mathrm{p}<0.01$ ) in RA patients compare to both groups. In addition Fn14 levels were higher in RA patients using biological drugs and seropositive subgroups (1042 \pm 1363 vs. $312 \pm 451 \mathrm{pg} / \mathrm{ml}, p<0.01$ and $674 \pm 101$ vs. $188 \pm 236 \mathrm{pg} / \mathrm{ml}$, respectively, $p<0.01$ ). Fn14 levels were correlated with disease duration $(r=0.38, p<0.01) . C D 163$ levels were similar in all groups. Although there was not any difference in cIMT measurements among groups, aortic stiffness was increased ( $p=0.03$ ), aortic strain and elasticity were decreased $(\mathrm{p}=0.03, \mathrm{p}=0.02)$ in RA patients compare to healthy controls. Multivariate analysis showed cardiovascular parameters were only associated with age.

Conclusions: In this study we observed impaired aortic parameters showing subclinic atherosclerosis in RA patients. At the same time, decreased levels of serum STWEAK and elevated levels of serum SFn14, were also shown in RA group. The difference in levels of serum soluble form of these biomarkers may be related to vascular damage in our RA patients who have low disease activity. However, further studies are needed to demonstrate association between atherosclerosis and these biomarkers in RA patients.

Disclosure of Interest: None declared

DOI: 10.1136/annrheumdis-2017-eular.2267

\section{AB1047 CAN THE ACR/EULAR 2010 CLASSIFICATION CRITERIA BE USED AS DIAGNOSTIC TOOL FOR RHEUMATOID ARTHRITIS IN REAL LIFE?}

N.F.L. Conijn ${ }^{1}$, D. Lopes Barreto ${ }^{1}$, T.M. Kuijper ${ }^{1}$, M. van der Steen $^{2}$, J. van der Kooij ${ }^{3}$, A.E.A.M. Weel ${ }^{1}$, J.M.W. Hazes ${ }^{4}$, M.R. KoK ${ }^{1} .{ }^{1}$ Rheumatology; ${ }^{2}$ Business intelligence; ${ }^{3}$ Planning and Control, Maasstad hospital; ${ }^{4}$ Rheumatology, Erasmus Medical Center, Rotterdam, Netherlands

Background: To date, due to the large variability in its clinical manifestations, early identification of rheumatoid arthritis (RA) relies on practice-based evidence. Moreover, this variation hampers the comparability and accurate stratification of the base population within and between RA trials. In 2010 the ACR/EULAR working group developed RA classification criteria that were primarily intended for research purposes. Despite its use in scientific settings, one can speculate on the effectiveness of these criteria when used in the routine clinical practice of diagnosing RA.

Objectives: In this study we aimed to investigate the degree of concordance between the diagnosis of RA in routine clinical practice and the ACR/EULAR 2010 classification criteria.

Methods: All patients who received a diagnosis of RA between 2010-2016 within our hospital were identified according to the financial diagnosis treatment combination (DTC) code, which corresponds to the ICD-10. Clinical and demographic data were extracted from our digital patient records of which $10 \%$ of the data were cross-checked by random selection. In retrospect we collected variables at time of RA diagnosis such as: number and type of swollen/painful joints, inflammatory markers, rheumatoid factor (RF), anti-citrullinated protein antibody (ACPA), disease duration and patients primary/secondary/tertiary diagnosis according to the rheumatologist. Additionally, all patients were classified according to the ACR/EULAR 2010 criteria for RA. The degree of concordance was determined by descriptive statistics.

Results: The database included 977 patients with a DTC RA of which 673 (69\%) had RA according to the rheumatologist. From the patients who were clinically diagnosed with RA, $463(69 \%)$ fulfilled the ACR/EULAR 2010 criteria (see figure 1), this is $47 \%$ of the total DTC RA patients. The majority of the population was female $(72 \%)$ and the mean age was 59 . A number of $161(24 \%)$ patients were diagnosed with RA according to the rheumatologists, but did not fulfil the ACR

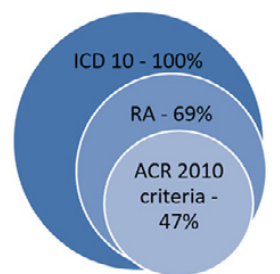

Fig. 1. Degree of concordance between RA identification and diagnosis, and classification criteria RA refers to the diagnosis RA made by the rheumatologist. As some patients with RA may have been incorrectly classified by ICD 10 , the circles are not completely overlapping. criteria. These patients had less inflammation, were more often RF and/or ACPA negative, and had less involved joints. About $5 \%$ of the data were missing. Conclusions: It can be concluded that the DTC codes are not the most reliable source of information in terms of the diagnosis. There is a discrepancy between the DTC code, the diagnosis according to the rheumatologist and the classification criteria. The degree of concordance between rheumatologist and the ACR criteria is comparable to the numbers described in literature. Since in our practice aspects of the ACR classification are used for diagnostic purposes, we will investigate factors that drive the specificity. Furthermore, reasons for the ICD-10/ DTC and final diagnostic mismatch is of great importance and will be studied as well. These factors will indicate opportunities on the use of the ACR/EULAR criteria in clinical practice. Acknowledgements: None

Disclosure of Interest: None declared

DOI: 10.1136/annrheumdis-2017-eular.5790

\section{AB1048 ULTRASOUND ASSESSMENT OF CARTILAGE THICKNESS IN PATIENTS WITH HAND OSTEOARTHRITIS}

O. Balueva ${ }^{1}$, A. Sarapulova ${ }^{2}$, O. Teplyakova ${ }^{2} .{ }^{1}$ Ultrasound Diagnosis, Medical Association "New hospital"; ${ }^{2}$ Urals State Medical University, Yekaterinburg, Russian Federation

Background: The destruction of cartilage is one of the basic mechanisms of the progression of hand osteoarthritis (HOA). Radiographic study indicates the thickness of the cartilage indirectly based on the degree of joint space narrowing (JSN). At the same time, the ultrasound (US) method allows to measure it directly. Objectives: To determine the thickness of the cartilage in the interphalangeal joints of patients with HOA and to correlate this rate with the degree of JSN, identified by radiologic imaging.c imaging.

Methods: 45 women at the age of $45-75$ years were included in the research. All of them were diagnosed of HOA according to the criteria of American College of Rheumatology (ACR). For each patient 8 proximal interphalangeal joints (PIP) were surveyed. The conventional radiographs (CRs) of each hand were performed for all patient in the standard frontal projection and the radiological changes were assessed using Kallman's method. Next, US investigation was carried out. The thickness of the cartilage was measured in the central part of the 2-5 proximal phalanx heads of both hands in the dorsal longitudinal view in the static image in gray- scale with the flexion position no more than 90 degrees. The cartilage is defined as a thin hypoechoic smooth layer, paralleling the contours of the articular surface. The thickness of the cartilage was defined as the distance between the subchondral bone and the surface layer of cartilage, which was the border between the cartilage and the joint cavity. In the presence of synovitis measurement of joint cartilage was carried out before the border and hypertrophic synovial if they differed in the degree of intensity of the US signal. The measurement of cartilage thickness was carried out by a conventional line, which was an exact perpendicular to the surface of the subchondral bone and parallel to the direction of US waves. Measurement was not carried out if visualization of the surface layer of cartilage was not possible. The control group consisted of 45 women $45-75$ years old without HOA, but with ultrasound investigation. Data were analyzed using Statistica 10.0 and are presented as mean (standard deviation), "odds ratio" coefficient (OR) and $x 2$ Pearson's correlation coefficient. Results: 360 joints of both hands were investigated with successful measurement of the cartilage thickness of $338(94.7 \%)$ joints. The average value of the cartilage in all PIP was $0.31(0.11) \mathrm{mm}$. The maximum thickness of the cartilage was obtained in the 2nd PIP joints - $0.34(0.12) \mathrm{mm}$ on the right hand and $0.36(0.14)$ $\mathrm{mm}$ on the left hand. For the control group results were $0.38(0.11) \mathrm{mm}$ and 0.37 $(0.13) \mathrm{mm}$ respectively. We have found a reliable relationship between the degree of JSN and cartilage thickness with the OR 1.849 (95\% confidence interval 1.198 $-2.855), x^{2}=7.772, p=0.0053$

Conclusions: US reduction of the cartilage thickness is a marker of cartilage loss which is correlated with the results of radiologic examination. This makes possible to use ultrasound as an alternative method of diagnosing HOA.

Disclosure of Interest: None declared

DOI: 10.1136/annrheumdis-2017-eular.6512

\section{AB1049 CLINICAL UTILITY OF AUTOANTIBODIES AGAINST EXTRACTABLE NUCLEAR ANTIGENS IN ROUTINE CARE: FREQUENCY OF REPEATED TEST REQUESTS AND DIAGNOSTIC VALUE OF ANTI-JO-1 (ANTI-HISTIDYL-TRNA SYNTHETASE)}

P. Jobanputra ${ }^{1}$, F. Malik ${ }^{2}$, E. Derrett-Smith ${ }^{1}$, T. Plant ${ }^{3}$, A. Richter ${ }^{4} .{ }^{1}$ Department of Rheumatology, Queen Elizabeth Hospital Birmingham, Birmingham; ${ }^{2}$ Department of Rheumatology, Epsom \& St. Helier University Hospitals NHS Trust, Carshalton; ${ }^{3}$ Department of Immunology, University of Birmingham; ${ }^{4}$ Department of Immunology, Queen Elizabeth Hospital Birmingham, Birmingham, United Kingdom

Background: High-throughput high-sensitivity ELISAs for autoantibodies associated with CTD, such as extractable nuclear antigens (ENA), are used widely. Anti-Jo-1 (anti-histidyl-tRNA synthetase), one of this panel, is believed to confer a poor prognosis due to an association with interstitial lung disease (ILD) and myositis. 
Objectives: To describe: the pattern of anti-ENA positive tests; frequency of repeated requests; stability and repeatability of anti-Jo- 1 tests; clinical characteristics of anti-Jo-1 + ves compared with controls; and diagnostic value of anti-Jo-1 for ILD.

Methods: All anti-ENA test requests, from any hospital department, between Jan 2013 and Dec 2014 were identified. Serum samples are screened for ENA (Quanta Lite ${ }^{\circledR}$ ENA profile, Inova Diagnostics) and positive samples have specific ENA antibodies levels quantified. Data from anti-Jo-1 positive patients and controls was extracted from electronic records allowing a minimum of 12 months after first test.

Results:

\begin{tabular}{lccc}
\hline & Jo-1 Positive $(\mathrm{n}=40)$ & Controls $(\mathrm{n}=80)$ & $\mathrm{P}$ value \\
\hline Age, mean years (range) & $53(19-86)$ & $52(17-87)$ & - \\
Sex (\% female) & $70 \%$ & $79 \%$ & 0.37 \\
Dead & $13 \%$ & $4 \%$ & 0.12 \\
Current or previous malignancy & $10 \%$ & $10 \%$ & 1.0 \\
Raynauds & $17.5 \%$ & $6.3 \%$ & 0.10 \\
Inflammatory arthritis & $20 \%$ & $19 \%$ & 1.0 \\
Clinical myositis diagnosis & $5 \%$ & $1.3 \%$ & 0.26 \\
CPK $>1000$ units/liter & $5 \%$ & $1.3 \%$ & 0.26 \\
Interstitial lung disease & $12.5 \%$ & $6 \%$ & 0.30 \\
CT chest done during study period & $17 / 40$ & $20 / 80$ & 0.06 \\
ANA ( $\geq 1: 100)$ & $18 / 38(47.4 \%)$ & $22 / 79(27.8 \%)$ & 0.06 \\
RF & $8 / 25(32 \%)$ & $12 / 44(27.3 \%)$ & 0.78 \\
CCP & $0 / 19(0 \%)$ & $3 / 33(9.1 \%)$ & 0.54 \\
Anti-dsDNA (Crithidia +ve) & $7.5 \%$ & $1.3 \%$ & 0.11 \\
Scl70 & $7.5 \%$ & $0 \%$ & $* \star$ \\
SSA Ro & $10 \%$ & $0 \%$ & $* \star$ \\
SSB/La & $10 \%$ & $0 \%$ & ${ }^{* \star}$ \\
RNP & $10 \%$ & $0 \%$ & $* \star$ \\
\hline
\end{tabular}

${ }^{*}$ Fisher's exact test, two tailed. ${ }^{*}$ Statistical analyses were not done on these comparisons as by definition controls were negative for ENA antibodies.

4009 samples from 3581 patients were tested. The first sample tested, chronologically, was designated test of interest. $616(17.2 \%)$ patients were anti-ENA screen +ve, and $40(1.1 \%)$ anti-Jo-1 +ve (>20 AU/mL). Anti-ENA tests were done more than once for $350 / 3581$ (9.8\%) patients (428/4009 (10.7\%) samples) and for $7 / 40(17.5 \%)$ of anti-Jo-1 +ve patients. The median interval between 1st and 2nd requests: 124 days (IQR 233 days). The Table shows data for anti-Jo-1 patients and randomly selected ENA -ve controls. The frequency of ILD, myositis and Raynaud's was comparable. Sensitivity and specificity of Jo-1 for ILD, a key feature of "anti-synthetase syndrome", were $50 \%(\mathrm{Cl} 19-81 \%)$ and $68 \%(\mathrm{Cl} 59-77 \%)$ respectively. Positive predictive value $12.5 \%$ (Cl 4 to $27 \%$ ) and negative predictive value $93.8 \%(\mathrm{Cl} 86-98 \%)$. Of patients with the highest anti-Jo1 titres ( $>40 \mathrm{AU} / \mathrm{mL}, 10 / 40$ patients, 25\%): 3 had ILD, 1 myositis and 2 had a malignancy (disseminated melanoma and CML). Bland-Altman plots show that anti-Jo-1 values remained stable when patients were re-tested at another time but re-testing available stored samples from +ve patients showed important variation (Figure).

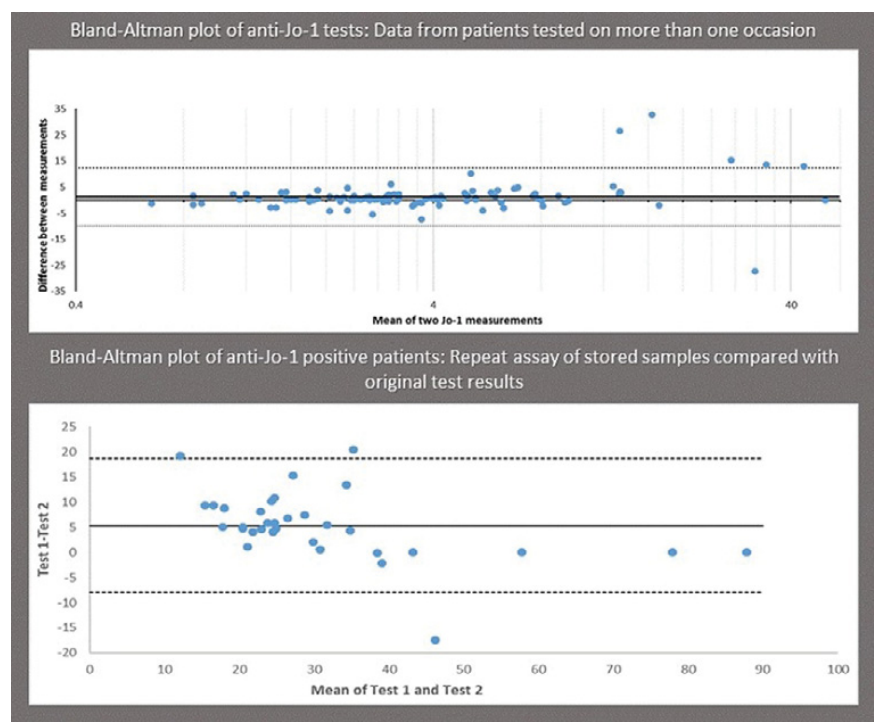

Conclusions: Anti-Jo-1 is uncommon in a heterogenous hospital population and is only weakly predictive for ILD. When tested repeatedly levels remain stable over many months. Repeated testing for anti-ENA is common and potentially unnecessary. Controls over repeated requests could yield cost savings.

Disclosure of Interest: None declared

DOI: 10.1136/annrheumdis-2017-eular.2939

\section{AB1050 COMPARATIVE STUDY BETWEEN ULTRASONOGRAPHIC ASSESSMENT AND CLINICAL EXAMINATION IN RHEUMATOID ARTHRITIS WITH OPTIMIZED BIOLOGICAL THERAPY AND NON BIOLOGICAL TREATMENT}

R. Martínez ${ }^{1}$, M. Fernández ${ }^{1}$, E. Rubio ${ }^{1}$, R. Menor Almagro ${ }^{1}$, B. Hernández ${ }^{2}$, J. Povedano ${ }^{2} .{ }^{1}$ Rheumatology, Hospital Universitario Virgen del Rocío;

${ }^{2}$ Rheumatology, Hospital Universitario Virgen Macarena, Sevilla, Spain

Background: In rheumatoid arthritis (RA), patients in low activity disease or clinical remission measured by disease activity indexs can present subclinical activity by ultrasound study. The ultrasonographic inflammation in examination of joints is an important predictive value of structural damage.

Objectives: The aim of our study was to describe subclinical ultrasonographics activity in patients with RA in low disease activity or clinical remission, with optimized biological therapy and non biological treatment.

Methods: Transversal and longitudinal study describing the ultrasonographic changes in gray scale and doppler in parallel with blind clinical evaluation. We included patients with RA according to ACR/EULAR classification criteria in low activity disease or remission measured by DAS28, under optimized biological therapy at least for 6 months and non biological treatment. They were sent by their usual clinician, making a random selection. They were evaluated in the same day by a rheumatologist and blind sonographer. VAS, VGP, VGM, HAQ, tender joints count, swollen joint count, CDAI, SDAI and DAS28 were evaluated. Regarding the ultrasound, were evaluated synovitis and doppler in 12 joints (wrists, second to fifth MCF and fifth bilateral MTF). The comparison between clinical examination and ultrasonography test was performed by the kappa index, with satisfactory value of $>0,6$

Results: A total of 69 patients were included, 35 with optimized biological therapy and 34 non biological treatment. The median optimization time was 12 months. The baseline characteristics only offered stadistical significance in swollen joint count and average time of disease (table 1).

The concordance study between clinical joint exploration and ultrasonographic examination showed a higher kappa index in patients with optimized biological therapy: 0,52 in gray scale and 0,40 in doppler. In patients without biological therapy showed an index kappa $=0.17$ by the gray scale test, and kappa index $=0.26$ by doppler.

Table 1

\begin{tabular}{lccc}
\hline Variable characteristics & $\begin{array}{c}\text { Non biological } \\
\text { treatment }\end{array}$ & $\begin{array}{c}\text { Optimized biological } \\
\text { therapy }\end{array}$ & $\mathrm{p}$ \\
\hline Age: average $\pm \mathrm{DE}$ (years) & $53,8 \pm 10$ & $54 \pm 11,7$ & 0,834 \\
Women (\%) & 73,5 & 65,7 & 0,498 \\
Time of diseases progression: & $55(34-116)$ & $120(84-139)$ & 0,003 \\
$\quad$ median (p25-p75) (months) & 71,3 & $81,80 \%$ & 0,544 \\
Rheumatoid factor + (\%) & 71,4 & 81,7 & 0,512 \\
Anti CCP + (\%) & $9,0 \pm 1,3$ & $8,1 \pm 1,4$ & 0,658 \\
Metotrexate dose: average \pm DE & $1,7 \pm 0,4$ & $1,5 \pm 0,4$ & 0,856 \\
Corticoids dose: average \pm DE & $1,0 \pm 0,2$ & $1,3 \pm 0,2$ & 0,309 \\
HAQ: average \pm DE & $0(0-1,5)$ & $1(0-2)$ & 0,200 \\
Tender joint count: median (p25-p75) & $0(0-1)$ & $1(0-2)$ & 0,013 \\
Swollen joint count: median (p25-p75) & $7,7 \pm 5,1$ & $6,8 \pm 5$ & 0,437 \\
CDAl: average \pm DE & $10,0 \pm 5,6$ & $10,0 \pm 6,5$ & 0,588 \\
SDAl: average \pm DE & & &
\end{tabular}

Conclusions: Our results show the existing discrepancy between the clinical examination and ultrasonographic test in patients in low disease activity/remission by DAS28, even more with the use of doppler. In the comparison of both groups we observed an increase in the difference in those who did not receive biological therapy. In patients with optimized biological teraphy, with higher swollen joint count in physical examination, kappa index was near of normality in grayscale. The detection of subclinical joint damage is often undertreated, showing ultrasound as a noninvasive technique of great help reducing joint damage.

Disclosure of Interest: None declared

DOI: 10.1136/annrheumdis-2017-eular.4406

\section{AB1051 ULTRASONOGRAPHY AND POWER DOPPLER ULTRASONOGRAPHY OF KNEE JOINT IN PATIENTS WITH HEPATITIS C VIRUS RELATED ARTHRITIS}

R. Elgohary ${ }^{1}$, A. Khalii ${ }^{2}$, G. Ragab ${ }^{3} .{ }^{1}$ Internal Medicine, Rheumatology \& Clinical Immunology subspecialty, Kasr Alainy; ${ }^{2}$ New Kasr El-Aini Teaching Hospital; ${ }^{3}$ Internal Medicine, Rheumatology \& Clinical Immunology subspecialty, Kasr Alainy, Cairo, Egypt

Background: HCV is a hepato-lymphotropic virus. Chronic arthritis is one of its extrahepatic manifestations ${ }^{1}$. The high frequency of rheumatoid factor (RF) positivity in $\mathrm{HCV}$ patients makes it difficult to differentiate between rheumatoid arthritis (RA) and HCV-related arthritis (HCVrA). An accurate and early diagnosis of HCVrA is important to avoid unnecessary immunosuppressive therapy ${ }^{2}$. Ultrasonography provides safe and quick access for the diagnosis of many rheumatic diseases.

Objectives: To illustrate ultrasonographic findings obtained in knee joints of patients with HCVrA \& to compare these findings with those obtained from knee joints of RA patients. 